\title{
On Certain Subclasses of Meromorphic Functions with Positive and Fixed Second Coefficients Involving the Liu-Srivastava Linear Operator
}

\author{
N. Magesh, ${ }^{1}$ N. B. Gatti, ${ }^{2}$ and S. Mayilvaganan ${ }^{3}$ \\ ${ }^{1}$ Research and P.G. Studies in Mathematics, Governt Arts College (Men), \\ Tamilnadu Krishnagiri 635001, India \\ 2 P.G. Studies in Mathematics, Government Science College, Karnataka Chitradurga 577501, India \\ ${ }^{3}$ Department of Mathematics, Adhiyamaan College of Engineering (Autonomous), \\ Tamilnadu Hosur 635109, India
}

Correspondence should be addressed to N. Magesh, nmagi_2000@yahoo.co.in

Received 23 December 2011; Accepted 16 February 2012

Academic Editor: S. Zhang

Copyright $@ 2012$ N. Magesh et al. This is an open access article distributed under the Creative Commons Attribution License, which permits unrestricted use, distribution, and reproduction in any medium, provided the original work is properly cited.

We introduce and study a subclass $\Sigma_{P}(\gamma, k, \lambda, c)$ of meromorphic univalent functions defined by certain linear operator involving the generalized hypergeometric function. We obtain coefficient estimates, extreme points, growth and distortion inequalities, radii of meromorphic starlikeness, and convexity for the class $\Sigma_{P}(\gamma, k, \lambda, c)$ by fixing the second coefficient. Further, it is shown that the class $\Sigma_{P}(\gamma, k, \lambda)$ is closed under convex linear combination.

\section{Introduction}

Let $\Sigma$ denote the class of functions of the form

$$
f(z)=z^{-1}+\sum_{n=1}^{\infty} a_{n} z^{n}
$$

which are analytic in the punctured unit disk

$$
\mathbb{U}^{*}:=\{z: z \in \mathbb{C}, 0<|z|<1\}=: \mathbb{U} \backslash\{0\} .
$$

Let $\Sigma_{\mathcal{S}}, \Sigma^{*}(\gamma)$ and $\Sigma_{K}(\gamma),(0 \leq \gamma<1)$ denote the subclasses of $\Sigma$ that are meromorphically univalent, meromorphically starlike functions of order $\gamma$, and meromorphically convex 
functions of order $\gamma$, respectively. Analytically, $f \in \Sigma^{*}(\gamma)$ if and only if, $f$ is of the form (1.1) and satisfies

$$
-\mathfrak{R}\left\{\frac{z f^{\prime}(z)}{f(z)}\right\}>\gamma, \quad z \in \mathbb{U},
$$

similarly, $f \in \Sigma_{K}(\gamma)$, if and only if, $f$ is of the form (1.1) and satisfies

$$
-\mathfrak{R}\left\{1+\frac{z f^{\prime \prime}(z)}{f^{\prime}(z)}\right\}>\gamma, \quad z \in \mathbb{U}
$$

and similar other classes of meromorphically univalent functions have been extensively studied by Aouf and Darwish [1], Aouf and Joshi [2], Mogra et al. [3], Uralegaddi and Ganigi [4], Uralegaddi and Somanatha[5], and Owa and Pascu [6].

Let $f, g \in \Sigma$, where $f(z)$ is given by (1.1) and $g(z)$ is defined by

$$
g(z)=z^{-1}+\sum_{n=1}^{\infty} b_{n} z^{n}
$$

Then the Hadamard product (or convolution) $f * g$ of the functions $f(z)$ and $g(z)$ is defined by

$$
(f * g)(z):=z^{-1}+\sum_{n=1}^{\infty} a_{n} b_{n} z^{n}=:(g * f)(z)
$$

Let $\Sigma_{P}$ be the class of functions of the form

$$
f(z)=z^{-1}+\sum_{n=1}^{\infty} a_{n} z^{n}, \quad a_{n} \geq 0
$$

that are analytic and univalent in $\mathbb{U}^{*}$.

For complex parameters $\alpha_{1}, \ldots, \alpha_{l}$ and $\beta_{1}, \ldots, \beta_{m}\left(\beta_{j} \neq 0,-1, \ldots ; j=1,2, \ldots, m\right)$ the generalized hypergeometric function ${ }_{l} F_{m}(z)$ is defined by

$$
\begin{aligned}
{ }_{l} F_{m}(z) \equiv{ }_{l} F_{m}\left(\alpha_{1}, \ldots \alpha_{l} ; \beta_{1}, \ldots, \beta_{m} ; z\right):=\sum_{n=0}^{\infty} \frac{\left(\alpha_{1}\right)_{n} \ldots\left(\alpha_{l}\right)_{n}}{\left(\beta_{1}\right)_{n} \ldots\left(\beta_{m}\right)_{n}} \frac{z^{n}}{n !} \\
\left(l \leq m+1 ; l, m \in \mathbb{N}_{0}:=\mathbb{N} \cup\{0\} ; z \in U\right),
\end{aligned}
$$

where $\mathbb{N}$ denotes the set of all positive integers and $(\theta)_{n}$ is the Pochhammer symbol defined by

$$
(\theta)_{n}=\frac{\Gamma(\theta+n)}{\Gamma(\theta)}= \begin{cases}1, & n=0 ; \theta \in \mathbb{C} \backslash\{0\} \\ \theta(\theta+1)(\theta+2) \cdot(\theta+n-1), & n \in \mathbb{N} ; \theta \in \mathbb{C}\end{cases}
$$


Corresponding to a function ${ }_{l} F_{m}\left(\alpha_{1}, \ldots \alpha_{l} ; \beta_{1}, \ldots, \beta_{m} ; z\right)$ defined by

$$
\mathcal{F}\left(\alpha_{1}, \ldots \alpha_{l} ; \beta_{1}, \ldots, \beta_{m} ; z\right):=z^{-1}{ }_{l} F_{m}\left(\alpha_{1}, \ldots \alpha_{l} ; \beta_{1}, \ldots, \beta_{m} ; z\right) .
$$

Liu and Srivastava [7] considered a linear operator $\mathscr{\ell}\left(\alpha_{1}, \ldots \alpha_{l} ; \beta_{1}, \ldots, \beta_{m}\right): \Sigma \rightarrow \Sigma$ defined by the following Hadamard product (or convolution):

$$
\begin{aligned}
\mathscr{H}\left(\alpha_{1}, \ldots \alpha_{l} ; \beta_{1}, \ldots, \beta_{m}\right) f(z) & =\mathcal{F}\left(\alpha_{1}, \ldots \alpha_{l} ; \beta_{1}, \ldots, \beta_{m} ; z\right) * f(z) \\
& =z^{-1}+\sum_{n=1}^{\infty}\left|\frac{\left(\alpha_{1}\right)_{n+1} \ldots\left(\alpha_{l}\right)_{n+1}}{\left(\beta_{1}\right)_{n+1} \ldots\left(\beta_{m}\right)_{n+1}}\right| \frac{a_{n} z^{n}}{(n+1) !},
\end{aligned}
$$

where, $\alpha_{i}>0,(i=1,2, \ldots l), \beta_{j}>0,(j=1,2, \ldots m), l \leq m+1 ; l, m \in \mathbb{N}_{0}=\mathbb{N} \cup\{0\}$. For notational simplicity, we use a shorter notations $\mathscr{H}_{m}^{l}\left[\alpha_{1}\right]$ for $\mathscr{H}\left(\alpha_{1}, \ldots \alpha_{l} ; \beta_{1}, \ldots, \beta_{m}\right)$ and

$$
\Gamma_{n}\left(\alpha_{1}\right)=\frac{\left(\alpha_{1}\right)_{n+1} \ldots\left(\alpha_{l}\right)_{n+1}}{\left(\beta_{1}\right)_{n+1} \ldots\left(\beta_{m}\right)_{n+1}} \frac{1}{(n+1) !}
$$

unless otherwise stated in the sequel. We note that the linear operator $H_{m}^{l}\left[\alpha_{1}\right]$ was earlier defined for multivalent functions by Dziok and Srivastava [8] and was investigated by Liu and Srivastava [7]. follows.

Making use of the operator $\mathscr{H}_{m}^{l}\left[\alpha_{1}\right]$, now we consider a subclass of functions in $\Sigma_{P}$ as

Definition 1.1. For $0 \leq \gamma<1$ and $k \geq 0$ and $0 \leq \lambda<1 / 2$, let $\Sigma(\gamma, k, \lambda)$ denote a subclass of $\Sigma$ consisting functions of the form (1.1) satisfying the condition that

$$
\begin{aligned}
- & \Re\left\{\frac{z\left(\mathscr{L}\left[\alpha_{1}\right] f(z)\right)^{\prime}+\lambda z^{2}\left(\mathscr{H}\left[\alpha_{1}\right] f(z)\right)^{\prime \prime}}{(1-\lambda) \mathscr{L}\left[\alpha_{1}\right] f(z)+\lambda z\left(\mathscr{L}\left[\alpha_{1}\right] f(z)\right)^{\prime}}+\gamma\right\} \\
& >k\left|\frac{z\left(\mathscr{L}\left[\alpha_{1}\right] f(z)\right)^{\prime}+\lambda z^{2}\left(\mathscr{L}\left[\alpha_{1}\right] f(z)\right)^{\prime \prime}}{(1-\lambda) \mathscr{H}\left[\alpha_{1}\right] f(z)+\lambda z\left(\mathscr{L}\left[\alpha_{1}\right] f(z)\right)^{\prime}}+1\right|, \quad z \in \mathbb{U}^{*},
\end{aligned}
$$

where $\mathscr{L}\left[\alpha_{1}\right] f(z)$ is given by (1.11). Furthermore, we say that a function $f \in \Sigma_{P}(\gamma, k, \lambda)$, whenever $f(z)$ is of the form (1.7).

We observe that, by specializing the parameters $l, m, \alpha_{1}, \ldots, \alpha_{l}, \beta_{1}, \ldots, \beta_{m}, k, \gamma$ and $\lambda$ the class leads to various subclasses. As for illustrations, we present some examples for the cases.

Example 1.2. If $l=2$ and $m=1$ with $\alpha_{1}=1, \alpha_{2}=1, \beta_{1}=1$ and $f(z)$ of the form (1.7), then we obtain the new subclass $\mathcal{M}_{P}(\gamma, k, \lambda)$ defined by

$$
-\mathfrak{R}\left\{\frac{z f^{\prime}(z)+\lambda z^{2} f^{\prime \prime}(z)}{(1-\lambda) f(z)+\lambda z f^{\prime}(z)}+\gamma\right\}>k\left|\frac{z f^{\prime}(z)+\lambda z^{2} f^{\prime \prime}(z)}{(1-\lambda) f(z)+\lambda z f^{\prime}(z)}+1\right|, \quad z \in \mathbb{U}^{*} .
$$


Example 1.3. For $l=2, m=1, \alpha_{1}=\delta+1, \beta_{1}=\alpha_{2}=1$, and $f(z)$ of the form (1.7), then we get the new subclass $\Phi_{P}^{\delta}(\gamma, k, \lambda)$ defined by

$$
-\mathfrak{R}\left\{\frac{z\left(\boldsymbol{\Phi}^{\delta} f(z)\right)^{\prime}+\lambda z^{2}\left(\boldsymbol{\Phi}^{\delta} f(z)\right)^{\prime \prime}}{(1-\lambda) \boldsymbol{\Phi}^{\delta} f(z)+\lambda z\left(\boldsymbol{\Phi}^{\delta} f(z)\right)^{\prime}}+\gamma\right\}>k\left|\frac{z\left(\boldsymbol{\Phi}^{\delta} f(z)\right)^{\prime}+\lambda z^{2}\left(\boldsymbol{\Phi}^{\delta} f(z)\right)^{\prime \prime}}{(1-\lambda) \boldsymbol{\Phi}^{\delta} f(z)+\lambda z\left(\boldsymbol{\Phi}^{\delta} f(z)\right)^{\prime}}+1\right|, \quad z \in \mathbb{U}^{*},
$$

where $\Phi^{\delta} f(z)=1 /\left(z(1-z)^{\delta+1}\right) * f(z)(\delta>-1)$, is the differential operator which was introduced by Ganigi and Uralegaddi [9]. Also we note that the class $\Phi_{P}^{\delta}(\gamma, k, \lambda)$ was introduced by Atshan and Kulkarni [10].

Example 1.4. For $l=2, m=1, \alpha_{2}=1$, and $f(z)$ of the form (1.7), then we obtain the new subclass $\mathcal{L}_{P}(\gamma, k, \lambda)$ defined by

$$
\begin{aligned}
- & \Re\left\{\frac{z\left(\mathcal{L}\left[\alpha_{1}, \beta_{1}\right] f(z)\right)^{\prime}+\lambda z^{2}\left(\mathcal{L}\left[\alpha_{1}, \beta_{1}\right] f(z)\right)^{\prime \prime}}{(1-\lambda) \mathcal{L}\left[\alpha_{1}, \beta_{1}\right] f(z)+\lambda z\left(\mathcal{L}\left[\alpha_{1}, \beta_{1}\right] f(z)\right)^{\prime}}+\gamma\right\} \\
& >k\left|\frac{z\left(\mathcal{L}\left[\alpha_{1}, \beta_{1}\right] f(z)\right)^{\prime}+\lambda z^{2}\left(\mathcal{L}\left[\alpha_{1}, \beta_{1}\right] f(z)\right)^{\prime \prime}}{(1-\lambda) \mathcal{L}\left[\alpha_{1}, \beta_{1}\right] f(z)+\lambda z\left(\mathcal{L}\left[\alpha_{1}, \beta_{1}\right] f(z)\right)^{\prime}}+1\right|, \quad z \in \mathbb{U}^{*},
\end{aligned}
$$

where the operator $\mathcal{L}\left[\alpha_{1} ; \beta_{1}\right] f(z)$ was introduced and studied by Liu and Srivastava [11] (see also $[12,13])$.

For the class $\Sigma_{P}(\gamma, k, \lambda)$, the following characterization was given by Magesh et al. [14].

Theorem 1.5. Let $f \in \Sigma_{P}$ be given by (1.7). Then $f \in \Sigma_{P}(\gamma, k, \lambda)$, if and only if,

$$
\sum_{n=1}^{\infty}(1+(n-1) \lambda)[n(k+1)+(k+\gamma)] \Gamma_{n}\left(\alpha_{1}\right) a_{n} \leq(1-\gamma)(1-2 \lambda)
$$

where $\Gamma_{n}\left(\alpha_{1}\right)$ is given by (1.12). yields

For a function defined by (1.7) and in the class $\Sigma_{P}(\gamma, k, \lambda)$, Theorem 1.5, immediately

$$
\Gamma_{1}\left(\alpha_{1}\right) a_{1} \leq \frac{(1-\gamma)(1-2 \lambda)}{(2 k+\gamma+1)}
$$

Hence we may take

$$
\Gamma_{1}\left(\alpha_{1}\right) a_{1}=\frac{(1-\gamma)(1-2 \lambda) c}{(2 k+\gamma+1)}, \quad 0<c<1
$$

Motivated by Aouf and Darwish [1], Aouf and Joshi [2], Ghanim and Darus [12], and Uralegaddi [15], we now introduce the following class of functions and use the similar techniques to prove our results. 
Let $\Sigma_{P}(\gamma, k, \lambda, c)$ be the subclass of $\Sigma_{P}(\gamma, k, \lambda)$ consisting functions in of the form

$$
f(z)=\frac{1}{z}+\frac{(1-\gamma)(1-2 \lambda) c}{(2 k+\gamma+1)} z+\sum_{n=2}^{\infty} \Gamma_{n}\left(\alpha_{1}\right) a_{n} z^{n}, \quad 0<c<1 .
$$

In this paper, coefficient estimates, extreme points, growth and distortion bounds, radii of meromorphically starlikeness and convexity are discussed for the class $\Sigma_{P}(\gamma, k, \lambda)$ by fixing the second coefficient. Further, it is shown that the class $\Sigma_{P}(\gamma, k, \lambda)$ is closed under convex linear combination.

\section{Main Results}

In our first theorem, we now find out the coefficient inequality for the class $\Sigma_{P}(\gamma, k, \lambda, c)$.

Theorem 2.1. Let the function $f(z)$ defined by (1.20). Then $f \in \Sigma_{P}(\gamma, k, \lambda, c)$, if and only if,

$$
\sum_{n=2}^{\infty}(1+(n-1) \lambda)[n(k+1)+(k+\gamma)] \Gamma_{n}\left(\alpha_{1}\right) a_{n} \leq(1-\gamma)(1-2 \lambda)(1-c) .
$$

The result is sharp.

Proof. By putting

$$
\Gamma_{1}\left(\alpha_{1}\right) a_{1}=\frac{(1-\gamma)(1-2 \lambda) c}{(2 k+\gamma+1)}, \quad 0<c<1,
$$

in (1.17), the result is easily derived. The result is sharp for the function

$$
f(z)=\frac{1}{z}+\frac{(1-\gamma)(1-2 \lambda) c}{(2 k+\gamma+1)} z+\frac{(1-\gamma)(1-2 \lambda)(1-c)}{(1+(n-1) \lambda)[n(k+1)+(k+\gamma)] \Gamma_{n}\left(\alpha_{1}\right)} z^{n}, \quad n \geq 2 .
$$

Corollary 2.2. If the function $f$ defined by (1.20) is in the class $\Sigma_{P}(\gamma, k, \lambda, c)$, then

$$
a_{n} \leq \frac{(1-\gamma)(1-2 \lambda)(1-c)}{(1+(n-1) \lambda)[n(k+1)+(k+\gamma)] \Gamma_{n}\left(\alpha_{1}\right)}, \quad n \geq 2 .
$$

The result is sharp for the function $f(z)$ given by (2.3).

Next we prove the following growth and distortion properties for the class $\Sigma_{P}(\gamma, k, \lambda, c)$.

Theorem 2.3. If the function $f(z)$ defined by (1.20) is in the class $\Sigma_{P}(\gamma, k, \lambda, c)$ for $0<|z|=r<1$, then one has

$$
\begin{aligned}
\frac{1}{r}- & \frac{(1-\gamma)(1-2 \lambda) c}{(2 k+\gamma+1)} r-\frac{(1-\gamma)(1-2 \lambda)(1-c)}{(1+\lambda)(3 k+\gamma+2)} r^{2} \leq|f(z)| \\
& \leq \frac{1}{r}+\frac{(1-\gamma)(1-2 \lambda) c}{(2 k+\gamma+1)} r+\frac{(1-\gamma)(1-2 \lambda)(1-c)}{(1+\lambda)(3 k+\gamma+2)} r^{2}
\end{aligned}
$$


The result is sharp for the function $f(z)$ given by

$$
f(z)=\frac{1}{z}+\frac{(1-\gamma)(1-2 \lambda) c}{(2 k+\gamma+1)} z+\frac{(1-\gamma)(1-2 \lambda)(1-c)}{(1+\lambda)(3 k+\gamma+2)} z^{2}
$$

Proof. Since $\Sigma_{P}(\gamma, k, \lambda, c)$, Theorem 2.1 yields

$$
\Gamma_{n}\left(\alpha_{1}\right) a_{n} \leq \frac{(1-\gamma)(1-2 \lambda)(1-c)}{(1+(n-1) \lambda)[n(k+1)+(k+\gamma)]}, \quad n \geq 2
$$

Thus, for $0<|z|=r<1$,

$$
\begin{aligned}
|f(z)| & \leq \frac{1}{|z|}+\frac{(1-\gamma)(1-2 \lambda) c}{(2 k+\gamma+1)}|z|+\sum_{n=2}^{\infty} \Gamma_{n}\left(\alpha_{1}\right) a_{n}|z|^{n} \\
& \leq \frac{1}{r}+\frac{(1-\gamma)(1-2 \lambda) c}{(2 k+\gamma+1)} r+r^{2} \sum_{n=2}^{\infty} \Gamma_{n}\left(\alpha_{1}\right) a_{n} \\
& \leq \frac{1}{r}+\frac{(1-\gamma)(1-2 \lambda) c}{(2 k+\gamma+1)} r+\frac{(1-\gamma)(1-2 \lambda)(1-c)}{(1+\lambda)(3 k+\gamma+2)} r^{2}, \\
|f(z)| & \geq \frac{1}{|z|}-\frac{(1-\gamma)(1-2 \lambda) c}{(2 k+\gamma+1)}|z|-\sum_{n=2}^{\infty} \Gamma_{n}\left(\alpha_{1}\right) a_{n}|z|^{n} \\
& \geq \frac{1}{r}-\frac{(1-\gamma)(1-2 \lambda) c}{(2 k+\gamma+1)} r-r^{2} \sum_{n=2}^{\infty} \Gamma_{n}\left(\alpha_{1}\right) a_{n} \\
& \geq \frac{1}{r}-\frac{(1-\gamma)(1-2 \lambda) c}{(2 k+\gamma+1)} r-\frac{(1-\gamma)(1-2 \lambda)(1-c)}{(1+\lambda)(3 k+\gamma+2)} r^{2} .
\end{aligned}
$$

Thus the proof of the theorem is complete.

Theorem 2.4. If the function $f(z)$ defined by (1.20) is in the class $\Sigma_{P}(r, k, \lambda, c)$ for $0<|z|=r<1$, then one has

$$
\begin{aligned}
\frac{1}{r^{2}} & -\frac{(1-\gamma)(1-2 \lambda) c}{(2 k+\gamma+1)}-\frac{(1-\gamma)(1-2 \lambda)(1-c)}{(1+\lambda)(3 k+\gamma+2)} r \\
& \leq\left|f^{\prime}(z)\right| \leq \frac{1}{r^{2}}+\frac{(1-\gamma)(1-2 \lambda) c}{(2 k+\gamma+1)}+\frac{(1-\gamma)(1-2 \lambda)(1-c)}{(1+\lambda)(3 k+\gamma+2)} r
\end{aligned}
$$

The result is sharp for the function $f(z)$ given by

$$
f(z)=\frac{1}{z}+\frac{(1-\gamma)(1-2 \lambda) c}{(2 k+\gamma+1)} z+\frac{(1-\gamma)(1-2 \lambda)(1-c)}{(1+\lambda)(3 k+\gamma+2)} z^{2}
$$


Proof. In view of Theorem 2.1, it follows that

$$
n \Gamma_{n}\left(\alpha_{1}\right) a_{n} \leq \frac{n(1-\gamma)(1-2 \lambda)(1-c)}{(1+(n-1) \lambda)[n(k+1)+(k+\gamma)]}, \quad n \geq 2
$$

Thus, for $0<|z|=r<1$ and making use of (2.11), we obtain

$$
\begin{aligned}
\left|f^{\prime}(z)\right| & \leq\left|\frac{-1}{z^{2}}\right|+\frac{(1-\gamma)(1-2 \lambda) c}{(2 k+\gamma+1)}+\sum_{n=2}^{\infty} n \Gamma_{n}\left(\alpha_{1}\right) a_{n}|z|^{n-1} \\
& \leq \frac{1}{r^{2}}+\frac{(1-\gamma)(1-2 \lambda) c}{(2 k+\gamma+1)}+r \sum_{n=2}^{\infty} n \Gamma_{n}\left(\alpha_{1}\right) a_{n} \\
& \leq \frac{1}{r^{2}}+\frac{(1-\gamma)(1-2 \lambda) c}{(2 k+\gamma+1)}+\frac{(1-\gamma)(1-2 \lambda)(1-c)}{(1+\lambda)(3 k+\gamma+2)} r \\
\left|f^{\prime}(z)\right| & \geq\left|\frac{-1}{z^{2}}\right|-\frac{(1-\gamma)(1-2 \lambda) c}{(2 k+\gamma+1)}-\sum_{n=2}^{\infty} n \Gamma_{n}\left(\alpha_{1}\right) a_{n}|z|^{n-1} \\
& \geq \frac{1}{r^{2}}-\frac{(1-\gamma)(1-2 \lambda) c}{(2 k+\gamma+1)}-r \sum_{n=2}^{\infty} n \Gamma_{n}\left(\alpha_{1}\right) a_{n} \\
& \geq \frac{1}{r^{2}}-\frac{(1-\gamma)(1-2 \lambda) c}{(2 k+\gamma+1)}-\frac{(1-\gamma)(1-2 \lambda)(1-c)}{(1+\lambda)(3 k+\gamma+2)} r .
\end{aligned}
$$

Hence the result follows.

Next, we will show that the class $\Sigma_{P}(\gamma, k, \lambda, c)$ is closed under convex linear combination.

Theorem 2.5. If

$$
f_{1}(z)=\frac{1}{z}+\frac{(1-\gamma)(1-2 \lambda) c}{(2 k+\gamma+1)} z
$$

and for $n \geq 2$

$$
f_{n}(z)=\frac{1}{z}+\frac{(1-\gamma)(1-2 \lambda) c}{(2 k+\gamma+1)} z+\sum_{n=2}^{\infty} \frac{(1-\gamma)(1-2 \lambda)(1-c)}{(1+(n-1) \lambda)[n(k+1)+(k+\gamma)] \Gamma_{n}\left(\alpha_{1}\right)} z^{n}
$$

Then $f \in \Sigma_{P}(\gamma, k, \lambda, c)$ if and only if it can expressed in the form

$$
f(z)=\sum_{n=1}^{\infty} \mu_{n} f_{n}(z)
$$

where $\mu_{n} \geq 0$ and $\sum_{n=1}^{\infty} \mu_{n} \leq 1$. 
Proof. From (2.13), (2.14), and (2.15), we have

$$
f(z)=\frac{1}{z}+\frac{(1-\gamma)(1-2 \lambda) c}{(2 k+\gamma+1)} z+\sum_{n=2}^{\infty} \frac{(1-\gamma)(1-2 \lambda)(1-c) \mu_{n}}{(1+(n-1) \lambda)[n(k+1)+(k+\gamma)] \Gamma_{n}\left(\alpha_{1}\right)} z^{n} .
$$

Since

$$
\begin{aligned}
& \sum_{n=2}^{\infty} \frac{(1-\gamma)(1-2 \lambda)(1-c) \mu_{n}}{(1+(n-1) \lambda)[n(k+1)+(k+\gamma)] \Gamma_{n}\left(\alpha_{1}\right)} \frac{(1+(n-1) \lambda)[n(k+1)+(k+\gamma)] \Gamma_{n}\left(\alpha_{1}\right)}{(1-\gamma)(1-2 \lambda)(1-c)} \\
& \quad=\sum_{n=2}^{\infty} \mu_{n}=1-\mu_{1} \leq 1,
\end{aligned}
$$

it follows from Theorem 1.5 that the function $f \in \Sigma_{P}(\gamma, k, \lambda, c)$. Conversely, suppose that $f \in \Sigma_{P}(\gamma, k, \lambda, c)$. Since

$$
a_{n} \leq \frac{(1-\gamma)(1-2 \lambda)(1-c)}{(1+(n-1) \lambda)[n(k+1)+(k+\gamma)] \Gamma_{n}\left(\alpha_{1}\right)}, \quad n \geq 2 .
$$

Setting

$$
\begin{gathered}
\mu_{n}=\frac{(1+(n-1) \lambda)[n(k+1)+(k+\gamma)] \Gamma_{n}\left(\alpha_{1}\right)}{(1-\gamma)(1-2 \lambda)(1-c)} a_{n}, \\
\mu_{1}=1-\sum_{n=2}^{\infty} \mu_{n} .
\end{gathered}
$$

it follows that

$$
f(z)=\sum_{n=1}^{\infty} \mu_{n} f_{n}(z) .
$$

This completes the proof of the theorem.

Theorem 2.6. The class $\Sigma_{P}(\gamma, k, \lambda, c)$ is closed under linear combination.

Proof. Suppose that the function $f$ be given by (1.20), and let the function $g$ be given by

$$
g(z)=\frac{1}{z}+\frac{(1-\gamma)(1-2 \lambda) c}{(2 k+\gamma+1)} z+\sum_{n=2}^{\infty}\left|b_{n}\right| z^{n}, \quad n \geq 2
$$

Assuming that $f$ and $g$ are in the class $\Sigma_{P}(\gamma, k, \lambda, c)$, it is enough to prove that the function $h$ defined by

$$
h(z)=\mu f(z)+(1-\mu) g(z), \quad 0 \leq \mu \leq 1,
$$


is also in the class $\Sigma_{P}(\gamma, k, \lambda, c)$. Since

$$
h(z)=\frac{1}{z}+\frac{(1-\gamma)(1-2 \lambda) c}{(2 k+\gamma+1)} z+\sum_{n=2}^{\infty}\left|a_{n} \mu+(1-\mu) b_{n}\right| z^{n}
$$

we observe that

$$
\sum_{n=2}^{\infty}(1+(n-1) \lambda)[n(k+1)+(k+\gamma)] \Gamma_{n}\left(\alpha_{1}\right)\left|a_{n} \mu+(1-\mu) b_{n}\right| \leq(1-\gamma)(1-2 \lambda)(1-c),
$$

with the aid of Theorem 2.1. Thus, $h \in \Sigma_{P}(\gamma, k, \lambda, c)$.

Next we determine the radii of meromorphically starlikeness and convexity of order $\delta$ for functions in the class $\Sigma_{P}(\gamma, k, \lambda, c)$.

Theorem 2.7. Let the function $f(z)$ defined by (1.20) be in the class $\Sigma_{P}(\gamma, k, \lambda, c)$, then

(i) $f$ is meromorphically starlike of order $\delta(0 \leq \delta<1)$ in the disk $|z|<r_{1}(\gamma, k, \lambda, c, \delta)$ where $r_{1}(\gamma, k, \lambda, c, \delta)$, is the largest value for which

$$
\begin{aligned}
& \frac{(3-\delta)(1-\gamma)(1-2 \lambda) c}{(2 k+\gamma+1)} r^{2}+\frac{(n+2-\delta)(1-\gamma)(1-2 \lambda)(1-c)}{(1+(n-1) \lambda)[n(k+1)+(k+\gamma)]} r^{n+1} \\
& \quad \leq(1-\delta), \quad n \geq 2,
\end{aligned}
$$

(ii) $f$ is meromorphically convex of order $\delta(0 \leq \delta<1)$ in the disk $|z|<r_{2}(\gamma, k, \lambda, c, \delta)$ where $r_{2}(\gamma, k, \lambda, c, \delta)$, is the largest value for which

$$
\frac{(3-\delta)(1-\gamma)(1-2 \lambda) c}{(2 k+\gamma+1)} r^{2}+\frac{n(n+2-\delta)(1-\gamma)(1-2 \lambda)(1-c)}{(1+(n-1) \lambda)[n(k+1)+(k+\gamma)]} r^{n+1} \leq(1-\delta), \quad n \geq 2 .
$$

Each of these results is sharp for function $f(z)$ given by (2.3).

Proof. It is enough to highlight that

$$
\left|\frac{z f^{\prime}(z)}{f(z)}+1\right| \leq 1-\delta, \quad|z|<r_{1} .
$$

Thus, we have

$$
\begin{aligned}
& \left|\frac{z f^{\prime}(z)}{f(z)}+1\right| \\
& =\left|\frac{-1 / z+((V)(E) c /(Q)) z+\sum_{n=2}^{\infty} n \Gamma_{n}\left(\alpha_{1}\right) a_{n} z^{n}+1 / z+((V)(E) c /(Q)) z+\sum_{n=2}^{\infty} \Gamma_{n}\left(\alpha_{1}\right) a_{n} z^{n}}{1 / z+((V)(E) c /(Q)) z+\sum_{n=2}^{\infty} \Gamma_{n}\left(\alpha_{1}\right) a_{n} z^{n}}\right|,
\end{aligned}
$$


where $(Q)$ denotes $(2 k+\gamma+1), V$ denotes $(1-\gamma)$, and $(E)$ denotes $(1-2 \lambda)$.

Hence (2.28) holds true if

$$
\begin{aligned}
& \frac{2(1-\gamma)(1-2 \lambda) c}{(2 k+\gamma+1)} r^{2}+\sum_{n=2}^{\infty}(n+1) \Gamma_{n}\left(\alpha_{1}\right) a_{n} r^{n+1} \\
& \quad \leq(1-\delta)\left[1-\frac{(1-\gamma)(1-2 \lambda) c}{(2 k+\gamma+1)} r^{2}-\sum_{n=2}^{\infty} \Gamma_{n}\left(\alpha_{1}\right) a_{n} r^{n+1}\right],
\end{aligned}
$$

or,

$$
\frac{(3-\delta)(1-\gamma)(1-2 \lambda) c}{(2 k+\gamma+1)} r^{2}+\sum_{n=2}^{\infty}(n+2-\delta) \Gamma_{n}\left(\alpha_{1}\right) a_{n} r^{n+1} \leq(1-\delta),
$$

and it follows that, from (2.1), we may take

$$
a_{n} \leq \frac{(1-\gamma)(1-2 \lambda)(1-c)}{(1+(n-1) \lambda)[n(k+1)+(k+\gamma)] \Gamma_{n}\left(\alpha_{1}\right)} \mu_{n}, \quad n \geq 2,
$$

where $\mu_{n} \geq 0$ and $\sum_{n=2}^{\infty} \mu_{n} \leq 1$.

For each fixed $r$, we choose the positive integer $n_{0}=n_{0}(r)$ for which

$$
\frac{(n+2-\delta)}{(1+(n-1) \lambda)[n(k+1)+(k+\gamma)]} \Gamma_{n}\left(\alpha_{1}\right) r^{n+1}
$$

is maximal. Then it follows that

$$
\sum_{n=2}^{\infty}(n+2-\delta) \Gamma_{n}\left(\alpha_{1}\right) a_{n} r^{n+1} \leq \frac{\left(n_{0}+2-\delta\right)(1-\gamma)(1-2 \lambda)(1-c)}{\left(1+\left(n_{0}-1\right) \lambda\right)\left[n_{0}(k+1)+(k+\gamma)\right]} r^{n_{0}+1} .
$$

Then $f$ is starlike of order $\delta$ in $0<|z|<r_{1}(\gamma, k, \lambda, c, \delta)$ provided that

$$
\frac{(3-\delta)(1-\gamma)(1-2 \lambda) c}{(2 k+\gamma+1)} r^{2}+\frac{\left(n_{0}+2-\delta\right)(1-\gamma)(1-2 \lambda)(1-c)}{\left(1+\left(n_{0}-1\right) \lambda\right)\left[n_{0}(k+1)+(k+\gamma)\right]} r^{n_{0}+1} \leq(1-\delta) .
$$

We find the value $r_{0}=r_{0}(k, c, \delta, n)$ and the corresponding integer $n_{0}\left(r_{0}\right)$ so that

$$
\frac{(3-\delta)(1-\gamma)(1-2 \lambda) c}{(2 k+\gamma+1)} r^{2}+\frac{\left(n_{0}+2-\delta\right)(1-\gamma)(1-2 \lambda)(1-c)}{\left(1+\left(n_{0}-1\right) \lambda\right)\left[n_{0}(k+1)+(k+\gamma)\right]} r^{n_{0}+1}=(1-\delta) .
$$

It is the value for which the function $f(z)$ is starlike in $0<|z|<r_{0}$. (ii) In a similar manner, we can prove our result providing the radius of meromorphic convexity of order $\delta(0 \leq \delta<1)$ for functions in the class $\Sigma_{P}(\gamma, k, \lambda, c)$, so we skip the details of the proof of (ii). 


\section{References}

[1] M. K. Aouf and H. E. Darwish, "Certain meromorphically starlike functions with positive and fixed second coefficients," Turkish Journal of Mathematics, vol. 21, no. 3, pp. 311-316, 1997.

[2] M. K. Aouf and S. B. Joshi, "On certain subclasses of meromorphically starlike functions with positive coefficients," Soochow Journal of Mathematics, vol. 24, no. 2, pp. 79-90, 1998.

[3] M. L. Mogra, T. R. Reddy, and O. P. Juneja, "Meromorphic univalent functions with positive coefficients," Bulletin of the Australian Mathematical Society, vol. 32, no. 2, pp. 161-176, 1985.

[4] B. A. Uralegaddi and M. D. Ganigi, "A certain class of meromorphically starlike functions with positive coefficients," Pure and Applied Mathematika Sciences, vol. 26, no. 1-2, pp. 75-81, 1987.

[5] B. A. Uralegaddi and C. Somanatha, "Certain differential operators for meromorphic functions," Houston Journal of Mathematics, vol. 17, no. 2, pp. 279-284, 1991.

[6] S. Owa and N. N. Pascu, "Coefficient inequalities for certain classes of meromorphically starlike and meromorphically convex functions," Journal of Inequalities in Pure and Applied Mathematics, vol. 4, no. 1, Article ID 17, 6 pages, 2003.

[7] J.-L. Liu and H. M. Srivastava, "Classes of meromorphically multivalent functions associated with the generalized hypergeometric function," Mathematical and Computer Modelling, vol. 39, no. 1, pp. 21-34, 2004.

[8] J. Dziok and H. M. Srivastava, "Classes of analytic functions associated with the generalized hypergeometric function," Applied Mathematics and Computation, vol. 103, no. 1, pp. 1-13, 1999.

[9] M. R. Ganigi and B. A. Uralegaddi, "New criteria for meromorphic univalent functions," Bulletin Mathématique de la Société des Sciences Mathématiques de la République Socialiste de Roumanie. Nouvelle Série, vol. 33(81), no. 1, pp. 9-13, 1989.

[10] W. G. Atshan and S. R. Kulkarni, "Subclass of meromorphic functions with positive coefficients defined by Ruscheweyh derivative. I," Journal of Rajasthan Academy of Physical Sciences, vol. 6, no. 2, pp. 129-140, 2007.

[11] J.-L. Liu and H. M. Srivastava, "A linear operator and associated families of meromorphically multivalent functions," Journal of Mathematical Analysis and Applications, vol. 259, no. 2, pp. 566-581, 2001.

[12] F. Ghanim and M. Darus, "On class of hypergeometric meromorphic functions with fixed second positive coefficients," General Mathematics, vol. 17, no. 4, pp. 13-28, 2009.

[13] D. Yang, "Certain convolution operators for meromorphic functions," Southeast Asian Bulletin of Mathematics, vol. 25, no. 1, pp. 175-186, 2001.

[14] N. Magesh, N. B. Gatti, and S. Mayilvaganan, “On certain subclasses of meromorphic functions with positive coefficients associated with Liu-Srivastava linear operator," Preprint.

[15] B. A. Uralegaddi, "Meromorphically starlike functions with positive and fixed second coefficients," Kyungpook Mathematical Journal, vol. 29, no. 1, pp. 64-68, 1989. 


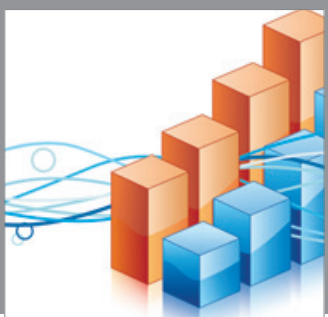

Advances in

Operations Research

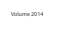

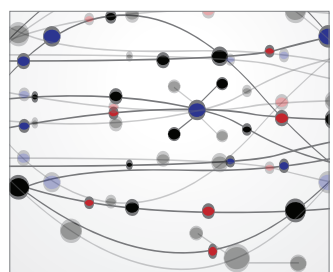

\section{The Scientific} World Journal
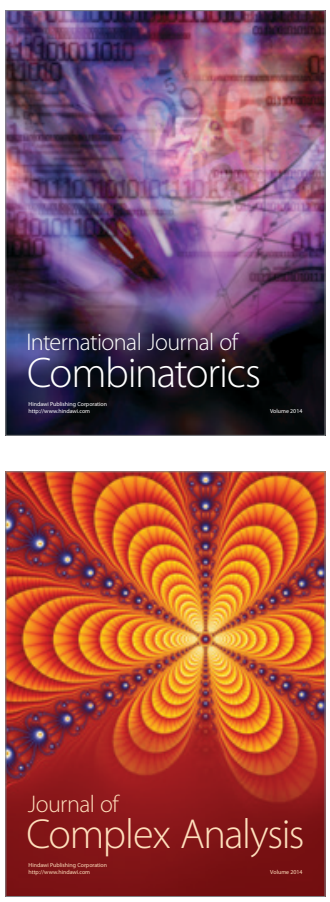

International Journal of

Mathematics and

Mathematical

Sciences
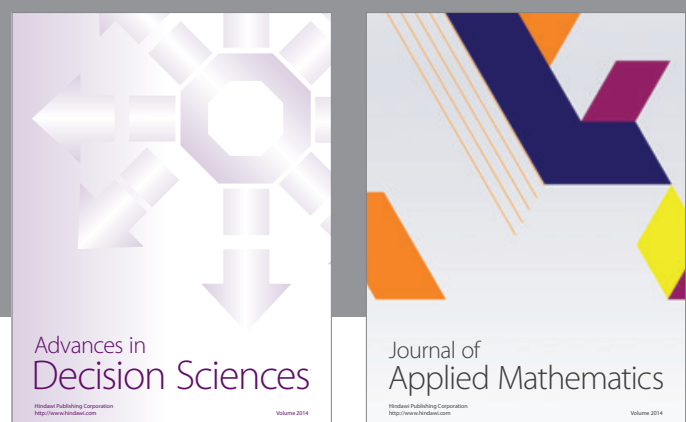

Journal of

Applied Mathematics
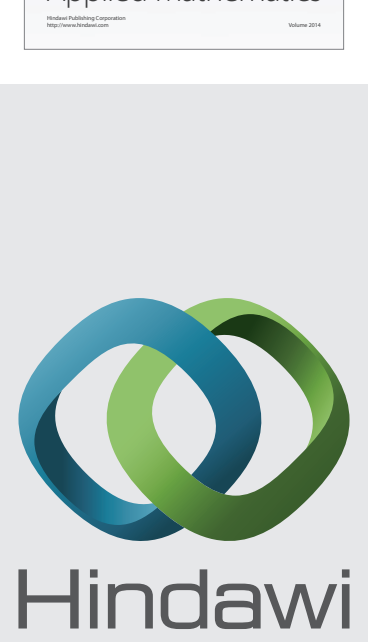

Submit your manuscripts at http://www.hindawi.com
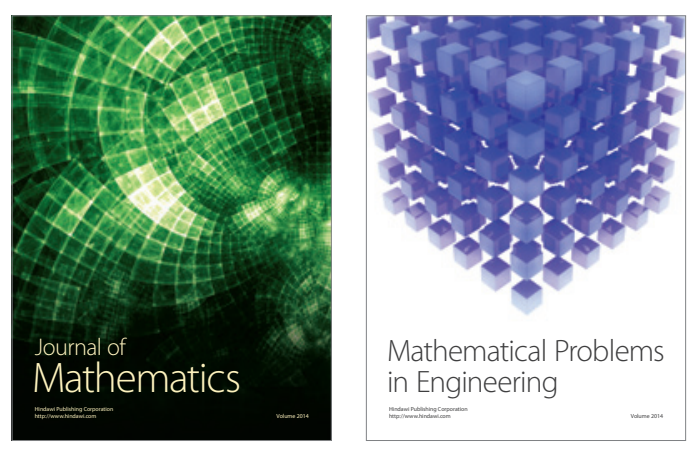

Mathematical Problems in Engineering
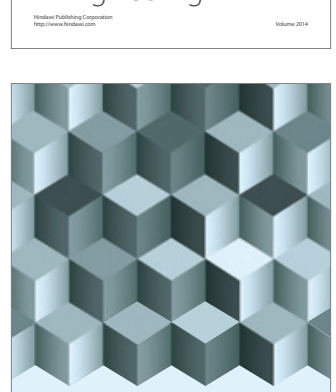

Journal of

Function Spaces
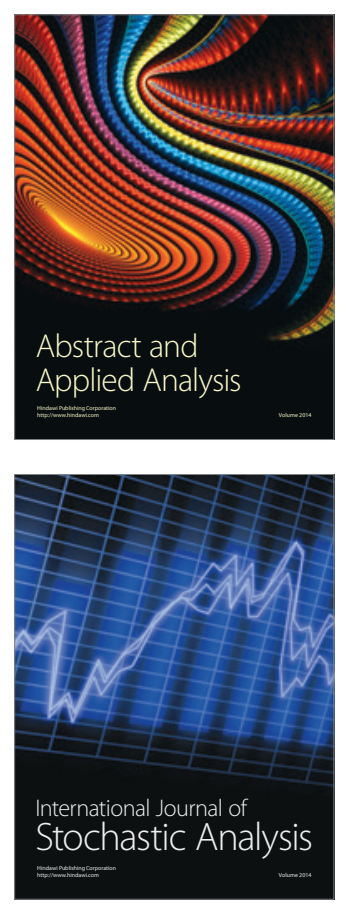

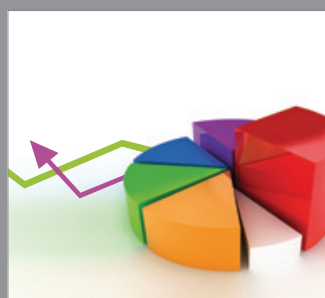

ournal of

Probability and Statistics

Promensencen
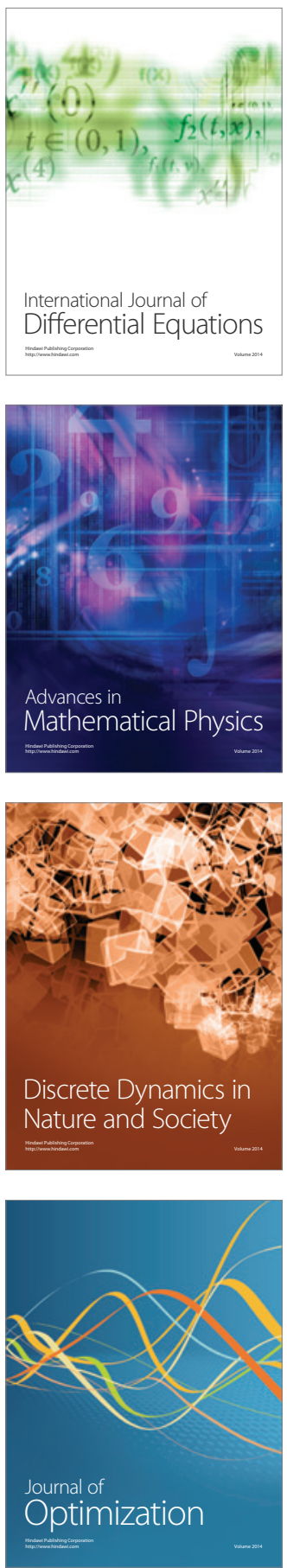